\title{
Susceptibility to klebsiella pneumonaie infection in collaborative cross mice is a complex trait controlled by at least three loci acting at different time points
}

Karin Vered ${ }^{1 \dagger}$, Caroline Durrant ${ }^{2 \dagger}$, Richard Mott $^{2}$ and Fuad A Iraqi ${ }^{1 *}$

\begin{abstract}
Background: Klebsiella pneumoniae (Kp) is a bacterium causing severe pneumonia in immunocompromised hosts and is often associated with sepsis. With the rise of antibiotic resistant bacteria, there is a need for new effective and affordable control methods; understanding the genetic architecture of susceptibility to Kp will help in their development. We performed the first quantitative trait locus (QTL) mapping study of host susceptibility to Kp infection in immunocompetent Collaborative Cross mice (CC). We challenged 328 mice from 73 CC lines intraperitoneally with $10^{4}$ colony forming units of Kp strain $\mathrm{K} 2$. Survival and body weight were monitored for 15 days post challenge. 48 of the CC lines were genotyped with 170,000 SNPs, with which we mapped QTLs.

Results: $C C$ lines differed significantly $(P<0.05)$ in mean survival time, between 1 to 15 days post infection, and broad sense heritability was 0.45 . Distinct QTL were mapped at specific time points during the challenge. A QTL on chromosome 4 was found only on day 2 post infection, and QTL on chromosomes 8 and 18, only on day 8 . By using the sequence variations of the eight inbred strain founders of the CC to refine QTL localization we identify several candidate genes.
\end{abstract}

Conclusion: Host susceptibility to $\mathrm{Kp}$ is a complex trait, controlled by multiple genetic factors that act sequentially during the course of infection.

Keywords: Klebsiella pneumoniae, Mouse model, Collaborative cross mice, Host susceptibility, QTL mapping, Candidate genes

\section{Background}

Klebsiella pneumoniae $(\mathrm{Kp})$ is a gram-negative enteric bacillus that is a common cause of nosocomial pneumonia after surgery, with significant associated morbidity and mortality $[1,2]$. With the rise of antibiotic resistance in bacteria, there is a need for alternative, effective and affordable control methods. Mouse models for Kp infection are well established, with a particular focus on the host immune response and the mechanisms induced to aid bacterial clearance [3]. It is well known that common inbred strains of mice often show differences in their genetic

\footnotetext{
* Correspondence: fuadi@post.tau.ac.il

${ }^{\dagger}$ Equal contributors

'Department of Clinical Microbiology and Immunology, Sackler Faculty of Medicine, Tel Aviv University, Tel Aviv, Israel
}

Full list of author information is available at the end of the article predisposition to infectious diseases. Genetic mapping has been used successfully to identify a number of murine loci conferring resistance to pathogenic diseases [4-6]. Consequently, the host response to Kp infection should also be amenable to genetic methods.

Although intercrosses between inbred lines of mice are powerful tools for mapping quantitative trait loci (QTL), with some important exceptions the genes underlying the QTLs remain unknown because the QTL intervals are too broad. Furthermore, because classical laboratory strains of mice originate from just a small sample of founders, they have a remarkably high level of shared ancestry largely contributed by the $M$. $m$. domesticus subspecies, and contain limited diversity. In contrast, wild-derived inbred strains encompasses genetic variation 
accumulated over about one million years. Thus each classical laboratory strain differs from the reference C57BL/6 J at about 4 million SNPs, whilst the wild-derived strains CAST/Ej and PWK/PhJ each differ at 17 million SNPs, and WSB/EiJ at 6 million [7].

Recognition of these problems resulted in the construction of the Collaborative Cross (CC), a general-purpose mouse resource to model complex traits $[8,9]$. The $C C$ is a population of recombinant inbred lines descended from eight divergent strains of mice, comprising five classical strains descended from M. m. domesticus (A/J, C57BL/6 J, 129S1/SvImJ, NOD/LtJ, NZO/HiLt)), combined with three wild-derived strains CAST/Ei (M. m. castaneus), PWK/PhJ (M. m. musculus), and WSB/EiJ (M. m. domesticus) [8]. Over 35 million SNPs segregate between the CC founders. Wild mice are constantly attacked by pathogens and the specificity and efficiency of their innate mechanisms of defense are under strong selective pressure.

We previously demonstrated the power of the immunecompetent CC lines for mapping QTL associated with host susceptibility to infection in a study of susceptibility to Aspergillus fumigatus [10]. The high genetic diversity in the CC population meant that we could map QTLs that involved contrasts of alleles segregating between the wildderived strains, and which would not have been visible in a cross between classical strains. These results suggested that host susceptibility to (Kp) infection should be amenable to investigation in the $\mathrm{CC}$ as well. In this study, we map susceptibility to $\mathrm{Kp}$ in the $\mathrm{CC}$, and identify QTL that are active in response to infection at distinct time points. Thus we have begun to dissect the underlying genetic and temporal architecture of Kp infection.

\section{Methods}

Mice

Mice were housed on hardwood chip bedding in opentop cages. They were kept on a 12-hour light/dark cycle and received distilled water and food ad libitum, before and during the challenge. All experimental protocols were approved by the Institutional Animal Care and Use Committee at TAU (IACUC), which adheres to Israeli guidelines, which follow the NIH/USA animal care and use protocols.

\section{Inbred mice}

Ten female mice aged eight weeks from each of the mouse strains BALB/CJ, DBA/2 J, C57BL/6 J and C3H/J were purchased from Harlan, Rehovot, Israel and used for testing.

\section{Collaborative cross mice}

Full details of the development of the CC colony are described in [9]. In this study 328 male (144 mice) and female (184 mice) CC mice from 73 lines (average 3-5 mice per line) at inbreeding generations of $8-17$ and between 8 to 12 weeks were used.

\section{Klebsiella pneumonia (Kp) inoculation}

Infection experiments with $\mathrm{Kp}$ were performed at the small animal facility at Sackler Faculty of Medicine, TelAviv University (TAU), Israel. Klebsiella pneumoniae strain K2 was provided by Izthak Ofek, Department of Clinical Microbiology and Immunology, Sackler Faculty of Medicine, Tel-Aviv University in the mid-log phase was obtained by growth in Luria broth (LB) composed of $10 \%$ Bacto Tryptone (Difco), 5\% yeast extract, and 5\% $\mathrm{NaCl}$ (pH 7.5) (Difco Laboratories, Detroit, MI) for $18 \mathrm{~h}$ at $37^{\circ} \mathrm{C}$ followed by inoculation into $\mathrm{LB}$ media for an additional $4 \mathrm{~h}$. K2 strain used in this study was isolated from human blood with full details is presented in previous reports [11,12] and was shown to be generally virulent in mice [13].

The Kp concentration in the broth was quantified by comparing absorbance at $595 \mathrm{~nm}$ with a standard curve. Bacteria were then diluted with sterile endotoxin-free saline to provide $\mathbf{1 0}^{\mathbf{4}}$ colony forming units ( $\mathrm{cfu}$ ) in a final volume of $0.2 \mathrm{ml}$. Bacterial numbers were confirmed by colony counts of LB agar plate dilutions after 24-h incubation at $37^{\circ} \mathrm{C}$. Animals were injected intraperitoneally (IP) with a final volume of $0.2 \mathrm{ml}$.

\section{Kp challenge}

Immunocompetent mice were challenged intraperitoneally with $10^{4} \mathrm{cfu}$ of $\mathrm{K} 2 \mathrm{Kp}$. Clinical assessment of susceptibility to infection during 15 days post challenge was based primarily on survival time. In addition, body weight and rectal body temperature, by a designed thermometer for small animal (NBT New Biotechnology Ltd. Jerusalem, Israel), was measured in survived animals at day 0, 2, 4, 8 and 14. Post-mortem testing for presence of $\mathrm{Kp}$ was by plating extracts from mouse tissues on MacConkey agar plates and counting cfu. Tail clips from all $\mathrm{CC}$ mice were saved as a DNA source.

\section{Postmortem testing of CFU Loads}

All succumbed and survived mice to infection were tested postmortem for $\mathrm{K} 2$ loads by plating extracts from different tissues for growth on LB media which solidified with $1.5 \%$ Bacto Agar and CFU counts method.

\section{Genotyping}

Representatives of 48 of the 73 CC lines used in this study had been genotyped previously (at WTCHG Oxford UK and at CISGen, UNC, USA), at 170000 SNPs, as described in [10]. Although the CC lines are not completely inbred, we have shown previously that using genotypes from a single representative from each line is sufficient for QTL mapping purposes. 


\section{Data analysis}

Data analysis was performed using the statistical software R ( $R$ Development Core Team 2009), including the $R$ package HAPPY.HBREM [14,15]. Survival data for the CC lines were converted into binary alive/dead phenotypes, one for each day of the trial. These binary phenotypes were analysed for the presence of QTLs by a two-stage process. Firstly, a logistic regression model was used to fit covariates, using the $\mathrm{R}$ function $\operatorname{glm}()$, and secondly the residuals from the model were used as the response variable for QTL mapping using linear regression, with the Bayesian random effects model HBREM [15] used to estimate the individual haplotype effects.

Two covariates significantly affected survival, sex and a batch effect. These effects were fitted via a logistic regression model where, for an individual of sex $i$ in batch group $\mathrm{j}$ with alive/dead status $\mathrm{y}_{\mathrm{ij}}$,

$$
\operatorname{In} \frac{\pi\left(y_{i j}\right)}{1-\pi\left(y_{i j}\right)}=\mu+\alpha+\gamma_{j}=\mu_{i j}
$$

Where $\pi\left(y_{i j}\right)$ is the probability of being dead, $\mu$ is the intercept, $\alpha_{i}$ is the effect of sex, and $\gamma_{j}$ is the effect of batch.

The genome of each $\mathrm{CC}$ line is a mosaic of the inbred founders, which we reconstructed using a hidden Markov Model (HMM) HAPPY [14] across the genotypes to compute probabilities of descent from the founders, setting the generation parameter to $g=7$. In CC line $k$ at SNP interval (locus) $L$, the HMM probability of descent from founder strain $s$ is denoted by $P_{L k(s)}$. The presence of a QTL at the locus $L$ is tested using a linear regression framework, in which the residual deviance from the mean probability of death $Y_{k}$ for an individual from line $k$ :

$$
\operatorname{In} \frac{\pi\left(y_{k}\right)}{1-\pi\left(y_{k}\right)} \mu+\sum_{s} P_{L K}(s) \beta_{s}
$$

where $\mu$ is the overall mean (incorporating any effects of batch and sex), and $\beta_{s}$ is the effect of founder haplotype $s$ at locus $L$.

The presence of a QTL is tested by comparing the fit of the model with that of a simpler submodel in which all the $\beta_{s}=0$ (the null hypothesis). Significance is reported as the $\log \mathrm{P}$, the negative $\log _{10}$ of the $\mathrm{p}$-value of the test of the null hypothesis, as computed by the $\mathrm{R}$ anova() function. Genomewide significance was estimated by permutation, where the $\mathrm{CC}$ line labels were permuted between the phenotypes. The median probability of death across replicates within each CC line was used in the QTL analysis. QTL effect sizes were estimated as the proportion of the log likelihood explained by the locus effects at the QTL.

\section{Estimation of heritability}

Heritability $\mathrm{H}^{2}$ was estimated as the proportion of phenotypic variation explained by differences between CC lines in the ANOVA, i.e. $H^{2}=V_{g} /\left(V_{g}+V_{e}\right)$. Full details on the heritability and Coefficient of Variation in CC mice were presented in our recent study [16].

\section{Estimation of QTL confidence intervals}

We estimated the CI for each QTL by simulation, using a similar approach as used in [10], to take into account local patterns of linkage disequilibrium.

\section{Testing sequence variation segregating between the CC founders}

We used the merge analysis [17] to test which variants under a QTL peak were compatible with the pattern of action at the QTL. We used the Sanger mouse genomes database [5] of sequence variants.

\section{Results}

Susceptibility to infection by Klebsiella pneumonia

We first compared the responses of four immune competent inbred strains (ten females from each of BALB/ CJ, DBA/2 J, C3H/HeJ and C57BL/6 J) and $73 \mathrm{CC}$ lines (3-7 mice per line) to infection with Kp to establish our assay was effective. Postmortem testing confirmed that all mice were infected with high cfu loads, indicating that death was caused by infection. All mice died during the infection, but with heritable variation in survival time (Additional file 1: Table S1). BALB/CJ mice were highly susceptible, $\mathrm{DBA} / 2 \mathrm{~J}$ and $\mathrm{C} 3 \mathrm{H} / \mathrm{HeJ}$ were highly resistant and C57BL/6 J was intermediate. BALB/CJ was significantly different from the three other strains (Additional file 1: Table S1). Mean survival time of BALB/CJ, C57BL/ $6 \mathrm{~J}, \mathrm{C} 3 \mathrm{H} / \mathrm{J}$ and $\mathrm{DBA} / 2 \mathrm{~J}$ was 2 days $(\mathrm{s} . \mathrm{d}=0.6), 2.8$ days $(\mathrm{sd}=0.56), 3.8$ days $(\mathrm{s} . \mathrm{d}=1.16)$, and 4 days $(\mathrm{s} . \mathrm{d}=1.88)$, respectively.

Body temperature and weight were shown not to be significantly changed during the infection, and have shown no correlations with survival time post infection, therefore were not presented and fully discussed in this report.

The 328 CC mice also responded variably with mean survival time between 1 to 12 days. Differences in survival between the $73 \mathrm{CC}$ lines were highly significant $(\mathrm{P}<0.0001)$. Figure 1 shows the percentage survival of a representative selection of susceptible, intermediate and resistant lines, Figure 2 shows the mean survival time (days) and standard deviation (s.d) of the representative selection CC lines and Additional file 2: Table S2 summarize the mean survival time (days) and standard deviation (s.d) of the entire studied CC lines. Although the variation between lines was highly significant, indicating that the response to infection was heritable, there was also considerable variability within some lines. Mice that survived past 


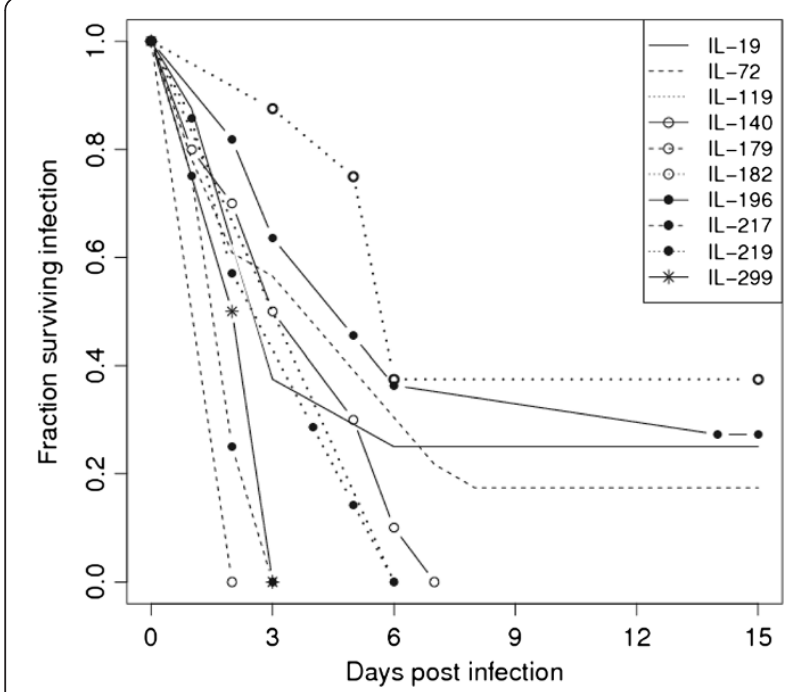

Figure 1 Survival curves of representative collaborative cross lines after intraperitoneal infection with Klebsiella pneumoniae.

the seventh day of infection tended to survive to the end of the experiment, suggesting this was a critical point in the disease progression.

The CC mice were assayed in 10 batches, two of which had different survival times, so we fitted a three-level batch covariate $(\mathrm{P}=6.84 \mathrm{e}-17)$. The number of inbreeding generations of each $\mathrm{CC}$ line had no effect on survival. However, sex was significant $(P=9.66 \mathrm{e}-05)$, with females less susceptible than males with mean survival days of 4.86 and 3.71, respectively. No correlation was observed between body weight (BW) and survival time (Pearson $\left.R^{2}=-0.07\right)$, or between body temperature (BT) and survival time $\left(R^{2}=0.053\right)$.

\section{QTL mapping}

We used a similar QTL mapping methodology to that previously employed in a study of host susceptibility to Aspergillosis in CC mice [10], except that we tested alive/dead survival status for QTLs at different time points, to identify early- and late-acting QTLs. Figure 3 and Table 1 summarize the three QTLs we found associated with host susceptibility to Kp infection at genomewide $\mathrm{E}<0.5(\mathrm{FDR}=8.3 \%)$. These QTL, named Kprl1 - Kprl3, (Klebsiella pneumonia resistant locus) were located on chromosomes 4, 8, and 18 respectively, $K p r l 1$ and 2 were mapped with $50 \%$ confidence intervals $(50 \% \mathrm{CIs}$ ) of 0.48 and $0.51 \mathrm{Mb}$ and $95 \%$ CIs of 7.03 and 5.44 Mb. Plots of the simulation results for the CIs are in Additional file 3: Figure S1. Kprl3 was mapped at 50\% genome wide significance (i.e. in $50 \%$ of genome scans with permuted $\mathrm{CC}$ line designations the genome-wide maximum $\log \mathrm{P}$ was less than that observed at $\mathrm{Kprl3}$ ), to a 50\% interval of $5.85 \mathrm{Mb}$ and a $95 \%$ interval of $18.06 \mathrm{Mb}$. However, the CI simulations around Kprl3 showed a different LD pattern focussed on the location of the original QTL, regardless of the location of the simulated locus (see Additional file 3: Figure S1). The two QTLs found on day 8 are clearly correlated. However, they show different patterns of haplotype effects so there does appear to be two different QTLs.

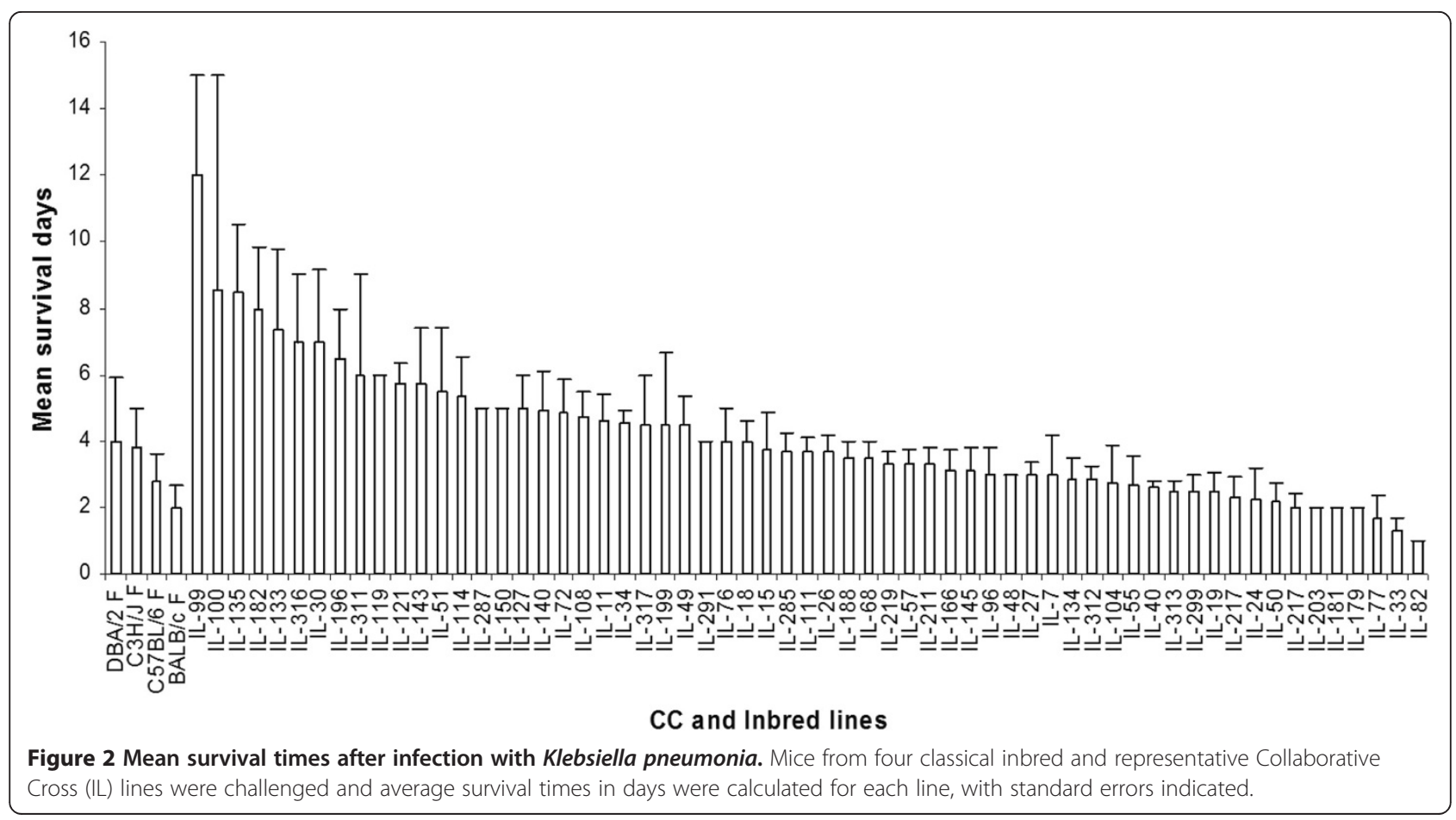




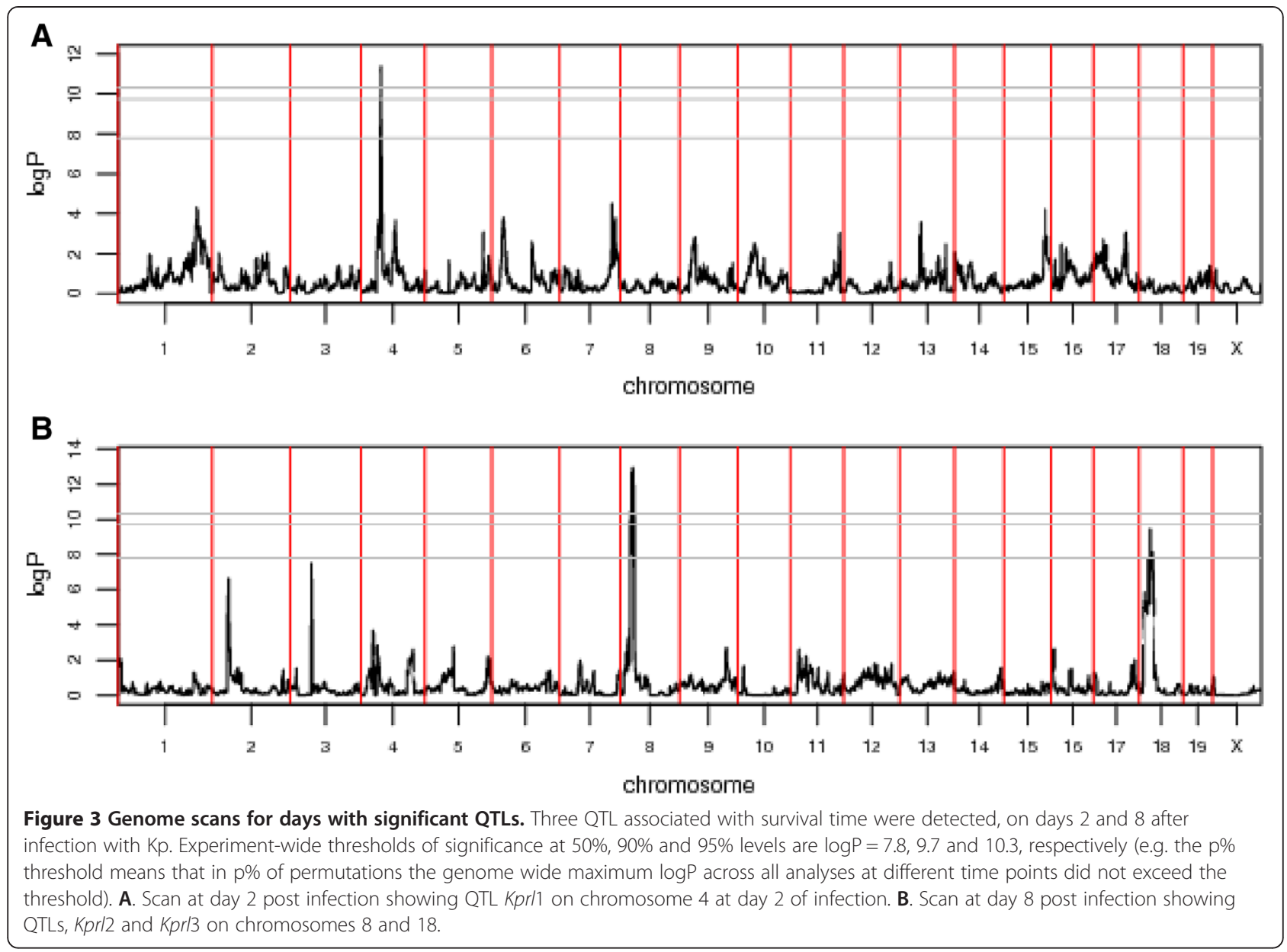

At each QTL we report the effect of each founder haplotype on survival time relative to WSB/EiJ, (Figure 4). All three loci showed complex patterns of haplotype effects, with the wild-derived strains playing a role but the inbred strains also contributing to the overall QTL effect. We also looked at the $\log \mathrm{P}$ at the QTL peak at each day of the experiment and plotted the temporal profile of each QTL through the experiment (Figure 5). This showed that all three QTLs are of short duration, one at the start of the experiment and two towards the end of the progression of the disease.

\section{Association analysis of sequence variations and} candidate genes

We used Merge Analysis [17] to impute and test association of sequence variants segregating between the $\mathrm{CC}$ founders within the QTLs. This takes advantage of the ancestry of the CC to infer the alleles of each CC line based on its genome mosaic (determined from its SNP genotypes) and sequence variation data in the founder strains. Where a QTL is caused by a single diallelic variant, we expect to have a high chance of testing a very tightly linked tagging SNP with the identical strain distribution pattern

Table 1 Positions of QTLs associated with susceptibility to Kp infection in 48 CC lines

\begin{tabular}{|c|c|c|c|c|c|c|c|c|c|c|c|}
\hline \multirow[t]{2}{*}{ QTL } & \multirow[t]{2}{*}{ Day } & \multirow[t]{2}{*}{ Chr } & \multirow[t]{2}{*}{$\log P$} & \multirow[t]{2}{*}{$\mathrm{H}^{2}$} & \multirow[t]{2}{*}{ Sig } & \multicolumn{2}{|c|}{$50 \% \mathrm{Cl}(\mathrm{Mb})$} & \multicolumn{2}{|c|}{$90 \% \mathrm{Cl}(\mathrm{Mb})$} & \multicolumn{2}{|c|}{$95 \% \mathrm{Cl}(\mathrm{Mb})$} \\
\hline & & & & & & Position & Width & Position & Width & Position & Width \\
\hline Kprl1 & 2 & 4 & 11.31 & 0.8 & 0.05 & $56.00-56.47$ & 0.48 & $54.27-58.28$ & 4.01 & $52.73-59.76$ & 7.03 \\
\hline Kprl2 & 8 & 8 & 12.85 & 0.83 & 0.05 & $31.72-32.23$ & 0.51 & $29.69-33.62$ & 3.93 & $29.07-34.52$ & 5.44 \\
\hline Kprl3 & 8 & 18 & 9.44 & 0.75 & 0.50 & $25.07-30.91$ & 5.85 & $19.89-36.42$ & 16.53 & $18.88-36.94$ & 18.06 \\
\hline
\end{tabular}

The chromosome $(\mathrm{Chr})$, negative $\log _{10} \mathrm{p}$-value $(\log \mathrm{P})$, heritability $\left(\mathrm{H}^{2}\right)$ and genome-wide significance level reached (Sig) are given, as well as the position and length of the $50 \%, 90 \%$ and $95 \%$ Cls relative to mouse genome build $\mathrm{mm} 9$. 
A

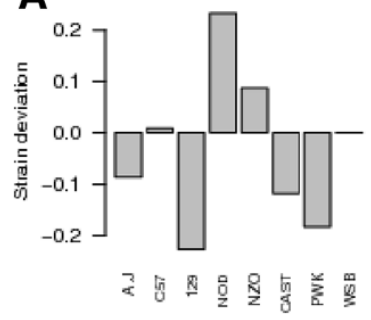

B

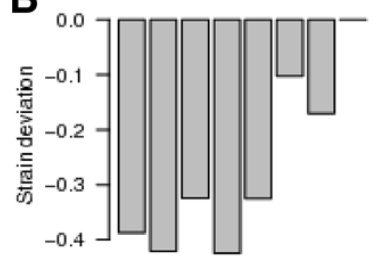

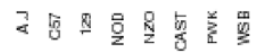

C

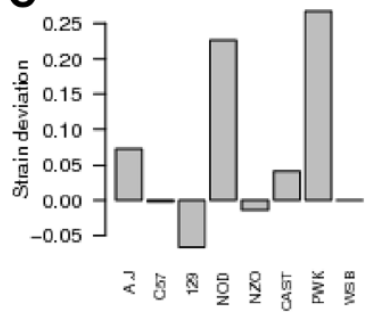

Figure 4 Estimated haplotype effects at QTLs for survival time following Kp infection. Effects are shown as deviations relative to WSB/EiJ, which is arbitrarily assigned the trait effect of 0 . The $x$-axis of each plot shows the founder strains; the $y$-axis shows the estimated haplotype effects of the CC founders. A. Kprl1, B. Kprl2, C. Kprl3.

in the founders as the causal variant. We also expect the merge analysis of such a SNP to have a higher logP-value than the 8-way haplotype test in the interval containing the variant, due to the reduction in the dimension of the test. If this is not observed, one possibility other than a false positive is that the QTL is caused by a combination of linked variants. It is also possible that an unknown and therefore untested sequence variant (for example an indel or CNV) that is not tagged by a known SNP could be responsible, although this is unlikely since most indels have similar SDP to a neighbouring SNP, and only a limited number of distinct strain distribution patterns are ever observed in the founders at each locus [18]. The merge analysis for Kprl1, 2 and 3 are shown in Figure 6A, B and $\mathrm{C}$ respectively.

The merge analysis of the three QTLs suggested several candidate causal genes with significant merge SNPs nearby. These are listed in Additional file 4: Table S3. Consistent with our earlier findings with Aspergillosis [10], the most significant merge analysis variants involved one wild-derived strain $v s$ the other 7 strains. In these QTLs we can exclude the great majority of variants from being causal. However, although the fraction of SNPs with the most associated SDP is small, these are evenly distributed across the QTL with $\log \mathrm{P}$ values that track those of the 8-way haplotype $\log \mathrm{P}$. Consequently many genes under the QTL will contain or be close to a variant with that SDP. Also, these SDPs pick out only a subset of the contrasts between the 8 haplotypes that are observed at the QTL peak. At Kprl2 and Kprl3, the merge SNPs with the best $\log \mathrm{Ps}$ are biallelic, despite the haplotype effects indicating a more complex situation. Presumably, none of the multiallelic SDPs matches the true situation closely enough to overcome the dimension penalty in the test. At Kprl1, there are virtually no significant merge SNPs at the QTL peak and none of them have an SDP matching the haplotype effects observed at the peak.

We classified the sequence variants under the QTLs according to whether their merge $\log \mathrm{P}$ was greater than the corresponding 8-way haplotype $\log \mathrm{P}$, and by their relationship to the genome annotation (Non Synonymous Coding, Synonymous Coding, 5' and 3' UTR, Splice Site or Intronic) (Additional file 4: Table S3).

The following genes that were identified by merge analysis are also strong candidates based on their known functions (see Additional file 4: Table S3): Kprl1 contained the candidates Ikbkap (Inhibitor of kappa light polypeptide gene enhancer in B-cells, kinase complex-associated protein). Actl7a and Actl7b (actin-like 7a and 7b), and Ctnnal1 (catenin alpha-like 1 gene), are involved in cell adhesion and cytoskeleton structure $[19,20]$. These are important in phagocytosis and for eliminating bacterial infection. Ctnnal1 is also important in the cell adhesion process $[21,22]$ and for capturing bacterial molecules to eliminate
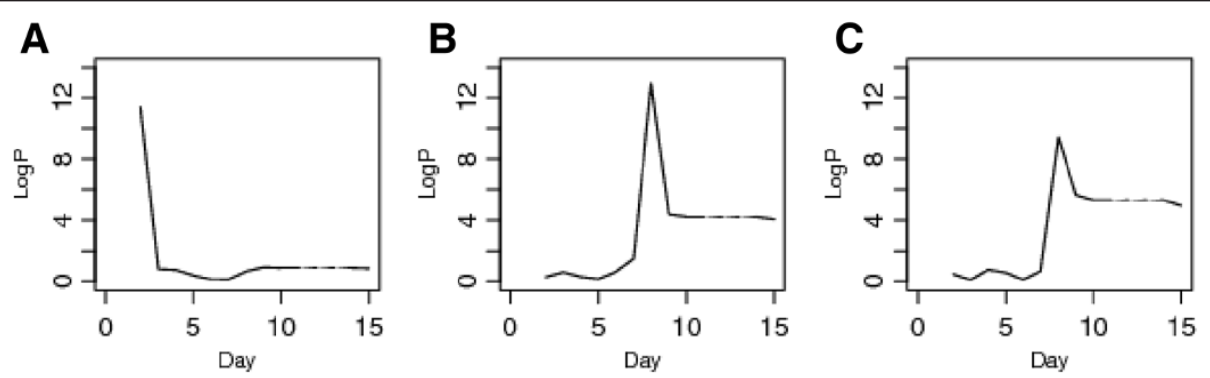

Figure 5 Plots of temporal profiles of the $\log \mathrm{P}$ at the QTLs over duration of experiment. A. Kprl1 on chromosome 4, B. Kprl2 on chromosome 8 and C. Kpr/3 on chromosome 18. 

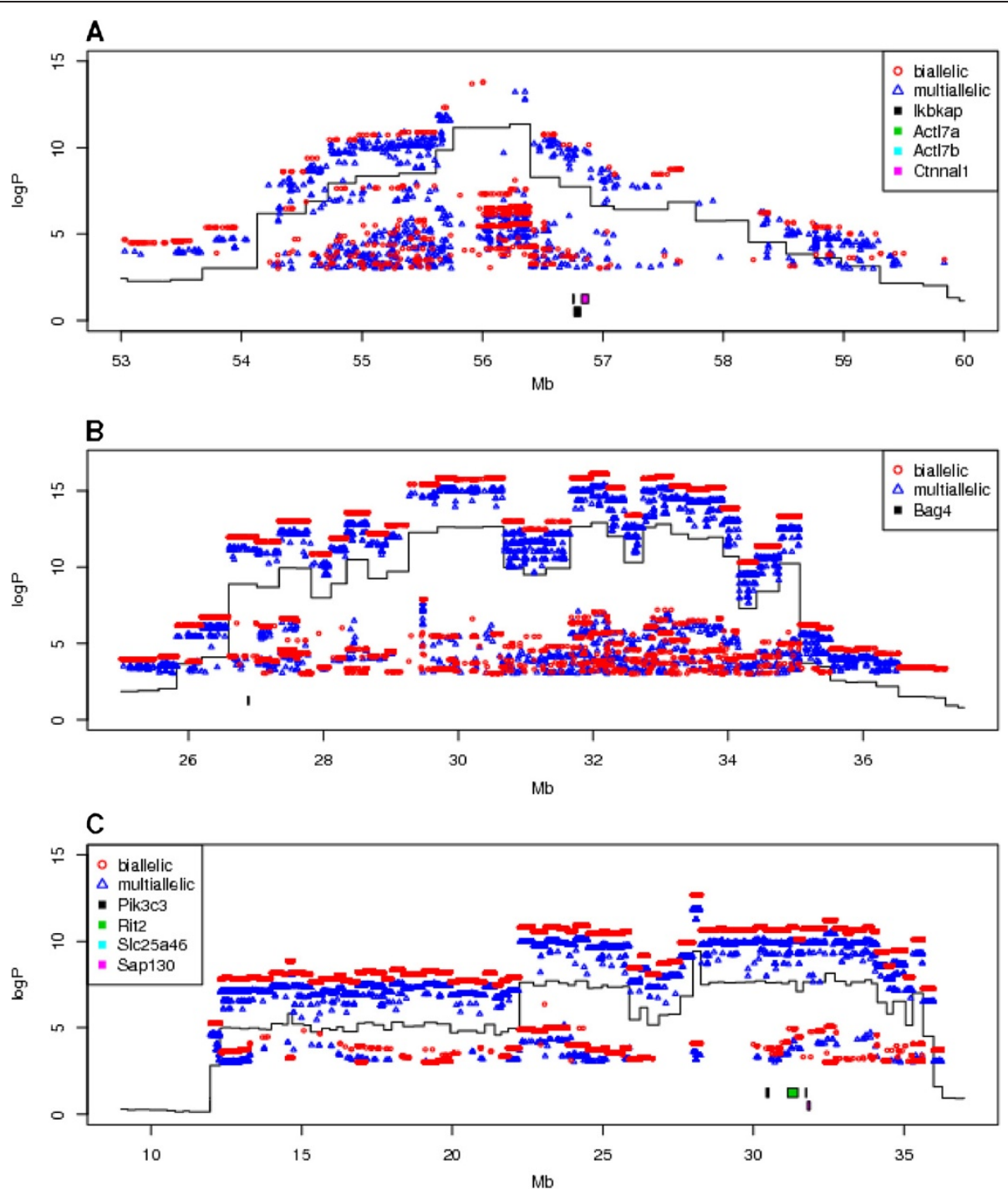

Figure 6 Merge analysis of sequence variants. The $x$-axis is genome location, $y$-axis is the logP of the test of association between locus and survival. The continuous black lines are sections of the genome scans in Figure 4. The blue dots are the results of merge analysis tests of sequence variants segregating in the 8 founders of the CC. For clarity, the great majority of variants with merge $\log P<1$ are not shown. Biallelic SNPs are in red circles, SNPs with more than two alleles (caused when alleles are unknown in one or more founders, which are then treated as unknown private alleles) are dark blue triangles. Image taken from the genome scan viewer http://mus.well.ox.ac.uk/gscandb. A. Kprl1 on chromosome 4; B. Kprl2 on chromosome 8 and C. Kprl3 on chromosome 18.

infection. Kprl2 contains the candidate gene Bag4 (BCL2associated athanogene 4) [23], which mediates cell-cell and cell-extracellular matrix interactions and this complex plays a role in host response to bacterial infection [24,25]. Homozygous mutant mice for Bag4 have enhanced cytokine responses and increased IL-6 production following TNF challenge i.e. septic shock [23]. Finally, Kprl3 contains the candidate genes Pik3c3 (phosphoinositide-3-kinase, class 3), Rit2 (ras-like without CAAX 2), Slc25a46 (solute carrier family 25, member 46) and Sap130 (Sin3A associated protein). Pik3c3 mice homozygous for a conditional allele activated in $\mathrm{T}$ cells exhibit impaired naive $\mathrm{T}$ cell homeostasis and mitophagy [26].

\section{Discussion}

In this study, we have used collaborative cross mice to identify QTLs, and suggest candidate genes contributing to, host response to $\mathrm{Kp}$ infection. The identification of genetic resistant factors to this disease will help of understanding why certain hosts succumb to the infection while others do not, and this will open new avenues for developing alternative control mechanisms to the infection.

The CC lines have more variable survival times compared to classical inbred strains, presumably due to the genetic diversity of the $\mathrm{CC}$ founders. $\mathrm{C} 3 \mathrm{H} / \mathrm{HeJ}$ mouse strain which is known be TLR4 deficient [27] showed to survive longer to the infection comparing to $\mathrm{BALB} / \mathrm{cJ}$ and $\mathrm{C} 57 \mathrm{BL} / 6 \mathrm{~J}$, but 
similar to DBA/2 J. However, based on the published results http://www.ncbi.nlm.nih.gov/gene/21898, TLR4 locus is located on chromosome 4 at $66 \mathrm{Mb}$ position, which is distal to Kprl1 locus, was not mapped with the tested traits and subsequently not involved or has little effect with this infection.

QTL mapping of survival times using survival regression was not successful, for despite the high heritability of survival time in the CC, no QTL was significant across all time points, indicating that the effects of any QTLs were transient. Instead, we defined a binary phenotype, alive or dead, for each day of the experiment and QTL mapping was performed separately for each day during the infection in order to detect transient QTLs. This novel analysis significantly improved power. We conclude that host susceptibility to $\mathrm{Kp}$ is a complex trait controlled by at least three loci acting at different times during the infection. To our knowledge, this is the first report using immunocompetent mouse strains to determine susceptibility to and map QTLs for Kp infection.

We compared these results with our previous analysis of the susceptibility of CC mice to aspergillosis [10], where the standard survival regression method, that assesses the overall effect of a locus throughout the experiment, was successful. The two infections have entirely different genetic architectures. Thus the method of analysis significantly affects the results, and the correct choice depends on the mode of action of the phenotype and possibly the immune status of the population. However, both studies show that the CC is well suited to the study of host response to infection, presumably because of the accumulation of genetic variation private to different subspecies, which presumably were exposed to differential selection pressures. Using the full genome sequence data of the eight founders of the CC [7], with the advantage of the merge analysis [17], it possible to refine the QTL map interval to small genomic regions and identify strong candidate genes. Searching for candidate genes within the fine mapped QTL regions revealed genes involve in T-cells regulations. One of the interesting candidate genes was found within Kprl2 QTL, Bag4 (BCL2-associated athanogene 4) [23], which mediates cell-cell and cell-extracellular matrix interactions and this complex plays a role in host response to bacterial infection [24,25]. Based on previous research, it was shown that homozygous mutant mice for Bag4 have enhanced cytokine responses and increased IL6 production following TNF challenge i.e. septic shock [23] (Kp is a major pathogenic cause of sepsis).

Identifying QTL act at different time points during challenge i.e. early v.s late phase was reported, earlier with trypanosome infection [28]. This is not surprising machines knowing that the host innate and adaptive immunity systems act at different time points during infection.
This report and those in [29-33] demonstrate the utility of the CC in the analysis of complex traits in mouse models of human disease. Our results underline the importance of the contribution of wild-derived alleles to the CC: the majority of the QTL we mapped involved a contrast with one of the three wild strains. The wild-derived founders may have different immune response mechanisms compared with the classical mouse strains. If so, we expect to identify novel response mechanisms to infectious diseases. This has two consequences. First, we can identify more genes using populations in which these variants segregate than in classical populations. Second, only sequence variants segregating in the CC founders that follow the same strain distribution pattern can be causal for the QTL. Therefore having a complete catalogue of sequence variants in the founders is of great utility. In effect, the combination of high-density genotypes in the $\mathrm{CC}$ and the genome sequences of the $\mathrm{CC}$ founders yield an approximate reconstruction of the sequence of each CC line as a mosaic of fragments of the founders' genomes. While this is currently limited to those regions of the genome that can be assembled from short-read sequence data, as sequencing technologies improve we expect to generate a complete catalogue of variation in the CC.

\section{Conclusion}

Our study has shown that host susceptibility to $\mathrm{Kp}$ is a complex trait, controlled by multiple genetic factors that act sequentially during the course of infection. The current study shows that even a modest number of lines [17] are useful with sufficient replication within each line. Nonetheless, in the near future over a hundred inbred and genotyped CC lines will be available to the research community, and using more lines will improve mapping resolution and power.

\section{Additional files}

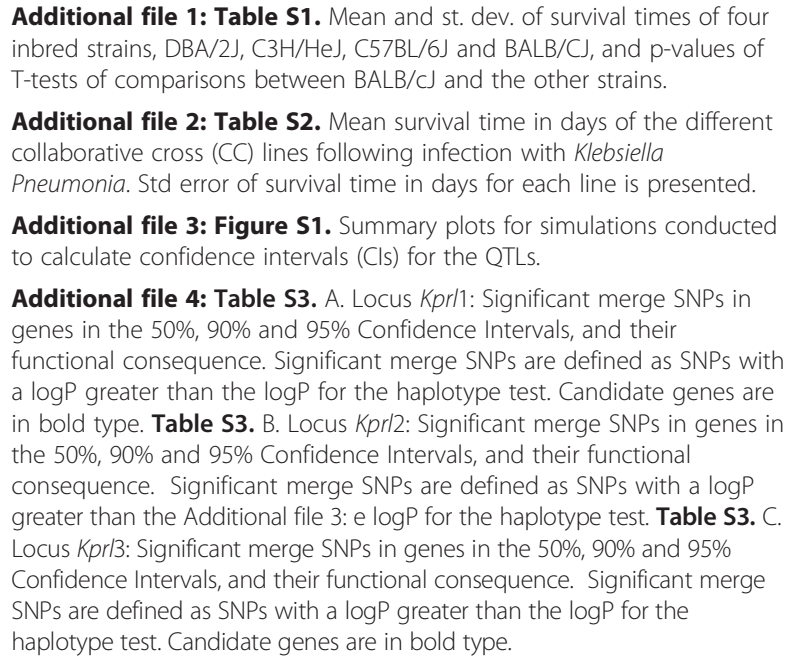

Additional file 1: Table S1. Mean and st. dev. of survival times of four inbred strains, DBA/2J, $\mathrm{C} 3 \mathrm{H} / \mathrm{HeJ}, \mathrm{C} 57 \mathrm{BL} / 6 \mathrm{~J}$ and BALB/CJ, and p-values of T-tests of comparisons between BALB/CJ and the other strains.

Additional file 2: Table S2. Mean survival time in days of the different collaborative cross (CC) lines following infection with Klebsiella Pneumonia. Std error of survival time in days for each line is presented.

Additional file 3: Figure S1. Summary plots for simulations conducted to calculate confidence intervals (Cls) for the QTLs.

Additional file 4: Table S3. A. Locus Kprl1: Significant merge SNPs in genes in the 50\%, 90\% and 95\% Confidence Intervals, and their functional consequence. Significant merge SNPs are defined as SNPs with a logP greater than the logP for the haplotype test. Candidate genes are in bold type. Table S3. B. Locus Kpr/2: Significant merge SNPs in genes in the 50\%, 90\% and 95\% Confidence Intervals, and their functional consequence. Significant merge SNPs are defined as SNPs with a $\log P$ greater than the Additional file 3: e logP for the haplotype test. Table S3. C. Locus Kpr/3: Significant merge SNPs in genes in the 50\%, 90\% and 95\% Confidence Intervals, and their functional consequence. Significant merge SNPs are defined as SNPs with a logP greater than the logP for the haplotype test. Candidate genes are in bold type. 


\section{Abbreviations}

CC: Collaborative cross; CTC: Complex trait consortium; QTL: Quantitative trait loci; TAU: Tel Aviv University; Kp: Klebsiella pneumonaie; Kprl: Klebsiella pneumonia resistant locus; Cls: confidence intervals; SNP: single nucleotide polymorphic; WTCHG: Wellcome Trsust Center for Human Genetics; UNC: University of North Carolina; cfu: Colony forming units.

\section{Competing interests}

The authors declare no potential conflict of interest with respect to financial or Non-financial competing interests, the authorship and/or publication of this article e.g. pharmaceutical stock ownership, consultancy.

\section{Authors' contributions}

KV participated in the design of the study, carried out the mice infection, participated in data analysis. CD participated in the data analyses and edits the first draft through the final submitted version. RM participated in the data analyses and edits the first draft through the final submitted version. FI participated in designing the study and in developing the first draft through the final submitted version of the manuscript. All authors read and approved the final manuscript.

\section{Acknowledgments}

The present work is part of a Ph.D. thesis by Karen Vered theses. The authors declare no competing financial interests or other associations that might pose a conflict of interest (e.g., pharmaceutical stock ownership, consultancy). This work was supported by the Wellcome Trust grants 085906/Z/08/Z, 075491/Z/04, Wellcome Trust core funding grant 090532/Z/ 09/Z, and core funding by Tel-Aviv University. SYSGENET COST action (BM0901) for providing financial support for short visits for Fuad A. Iraqi to Wellcome Trust Human Genome Center for conducting data and QTL analysis. The authors thank Prof. Itzhak Ofek for providing the Kp (K2 strain) bacteria and for his intellectual input to the study.

\section{Author details}

'Department of Clinical Microbiology and Immunology, Sackler Faculty of Medicine, Tel Aviv University, Tel Aviv, Israel. ${ }^{2}$ Wellcome Trust Centre for Human Genetics, University of Oxford, Oxford OX3 7BN, UK.

Received: 7 May 2014 Accepted: 24 September 2014

Published: 6 October 2014

\section{References}

1. Cross A, Allen JR, Burke J, Ducel G, Harris A, John J, Johnson D, Lew M, MacMillan B, Meers P: Nosocomial infections due to Pseudomonas aeruginosa: review of recent trends. Rev Infect Dis 1983, 5(Suppl):S837-S845.

2. Kreger BE, Craven DE, Carling PC, McCabe WR: Gram-negative bacteremia. III. Reassessment of etiology, epidemiology and ecology in 612 patients. Am J Med 1980, 68:332-343.

3. Franklin GA, Scott MJ, Patel M, Hoth JJ, Peyton JC, Cheadle WG: A novel model of pneumonia from intraperitoneal injection of bacteria. Am $J$ Surg 2003, 186(5):493-499.

4. Iraqi F, Clapcot S, Kuman P, Heley C, Kemp SJ, Teale AJ: Fine mapping of trypanosomiasis resistance QTLs in mice using advanced intercross lines. Mamm Genome 2000, 11:645-648.

5. Iraqi F, Behnke J, Menge D, Lowe A, Teale AJ, Gibson JP, Baker LR, Wakelin $D R$ : Chromosomal regions controlling resistance to gastro-intestinal nematode infections in mice. Mamm Genome 2003, 14:184-191.

6. Hernandez-Valladares M, Naessens J, Gibson JP, Musoke AJ, Rihet P, Ole-MoiYoi OK, Iraqi FA: Confirmation and dissection of QTL controlling resistance to malaria in mice. Mamm Genome 2004, 15:390-398.

7. Keane TM, Goodstadt L, Danecek P, White MA, Wong K, Yalcin B, Heger A, Agam A, Slater G, Goodson M, Furlotte NA, Eskin E, Nellåker C, Whitley H, Cleak J, Janowitz D, Hernandez-Pliego P, Edwards A, Belgard TG, Oliver PL, McIntyre RE, Bhomra A, Nicod J, Gan X, Yuan W, van der Weyden L, Steward CA, Bala S, Stalker J, Mott R, et al: Mouse genomic variation and its effect on phenotypes and gene regulation. Nature 2011, 477(7364):289-294.

8. Churchill GA, Airey DC, Allayee H, Angel JM, Attie AD, Beatty J, Beavis WD, Belknap JK, Bennett B, Berrettini W, Bleich A, Bogue M, Broman KW, Buck KJ, Buckler E, Burmeister M, Chesler EJ, Cheverud JM, Clapcote S, Cook MN, Cox RD, Crabbe JC, Crusio WE, Darvasi A, Deschepper CF, Doerge RW, Farber CR, Forejt J, Gaile D, Garlow SJ, et al: The Collaborative Cross, a community resource for the genetic analysis of complex traits. Nat Genet 2004, 36:1133-1137.

9. Iraqi FA, Churchill G, Mott R: The Collaborative Cross, developing a resource for mammalian systems genetics: a status report of the Wellcome Trust cohort. Mamm Genome 2008, 19(6):379-381.

10. Durrant C, Tayem H, Yalcin B, Cleak J, Goodstadt L, de Villena FP, Mott R Iraqi FA: Mapping QTL associated with host susceptibility to Aspergillus fumigatus infection in the Collaborative Cross mouse resource population. Genome Res 2011, 21(8):1239-1248.

11. Ofek I, Kabha K, Athamna A, Frankel G, Wozniak DJ, Hasty LD, Ohman ED: Genetic exchange of determinants for capsular polysaccharide biosynthesis between Klebsiella pneumoniae strains expressing serotypes K2 and K21a. Infect Immun 1993, 61:4208-4216.

12. Cryz SJ, Mortimer PM, Mansfield V, Germanier R: Seroepidemiology of Klebsiella bacteremic isolates and implications for vaccine development. J Clin Microbiol 1986, 23:687-690.

13. Mizuta K, Ohta M, Mori M, Hasegawa T, Nakashima I, Kato N: Virulence for mice of Klebsiella strains belonging to the 01 group: relationship to their capsular (K) types. Infect Immun 1983, 40:56-61.

14. Mott R, Talbot CJ, Turri MG, Collins AC, Flint J: A method for fine mapping quantitative trait loci in outbred animal stocks. Proc Natl Acad Sci U S A 2000, 97(23):12649-12654.

15. Durrant C, Mott R: Bayesian Quantitative Trait Locus mapping using inferred haplotypes. Genetics 2010, 184:839-852.

16. Iraqi FA, Atamni H, Dorman A, Salymah Y, Tomlinson I, Nashif A, Shusterman A, Weiss E, Houri-Haddad Y, Mott R, Soller M: The Collaborative Cross mouse reference population is fulfilling its promise as a source of wide genetic variation and high resolution QTL mapping. Mamm Genome 2014, 25(3):109-119.

17. Yalcin B, Flint J, Mott R: Using progenitor strain information to identify quantitative trait nucleotides in outbred mice. Genetics 2005, 171:673-681.

18. Yalcin B, Fullerton J, Miller S, Keays DA, Brady S, Bhomra A, Jefferson A, Volpi E, Copley RR, Flint J, Mott R: Unexpected complexity in the haplotypes of commonly used inbred strains of laboratory mice. Proc Natl Acad Sci U S A 2004, 101(26):9734-9739.

19. Chadwick BP, Mull J, Helbling LA, Gill S, Leyne M, Robbins CM, Pinkett HW, Makalowska I, Maayan C, Blumenfeld A, Axelrod FB, Brownstein M, Gusella JF, Slaugenhaupt $S A$ : Cloning, mapping, and expression of two novel actin genes, actin-like-7A $(A C T L 7 A)$ and actin-like-7B $(A C T L 7 B)$, from the familial dysautonomia candidate region on 9q31. Genomics 1999, 58(3):302-309.

20. Boeda B, Knowles PP, Briggs DC, Murray-Rust J, Soriano E, Garvalov BK, McDonald NQ, Way M: Molecular recognition of the Tes LIM2-3 domains by the actin-related protein Arp7A. J Biol Chem 2011, 286(13):11543-11554.

21. Ko MS, Kitchen JR, Wang $X$, Threat TA, Wang $X$, Threat TA, Wang $X$ Hasegawa A, Sun T, Grahovac MJ, Kargul GJ, Lim MK, Cui Y, Sano Y, Tanaka T, Liang Y, Mason S, Paonessa PD, Sauls AD, DePalma GE, Sharara R, Rowe LB, Eppig J, Morrell C, Doi H: Large-scale cDNA analysis reveals phased gene expression patterns during preimplantation mouse development. Development 2000, 127(8):1737-1749.

22. Skarnes WC, Rosen B, West AP, Koutsourakis M, Bushell W, lyer V, Mujica AO, Thomas M, Harrow J, Cox T, Jackson D, Severin J, Biggs P, Fu J, Nefedov M, de Jong PJ, Stewart AF, Bradley A: A conditional knockout resource for the genome-wide study of mouse gene function. Nature 2011, 474(7351):337-342.

23. Rahman P, Huysmans RD, Wiradjaja F, Gurung R, Ooms LM, Sheffield DA, Dyson JM, Layton MJ, Sriratana A, Takada H, Tiganis T, Mitchell CA: Silencer of death domains (SODD) inhibits skeletal muscle and kidney enriched inositol 5-phosphatase (SKIP) and regulates phosphoinositide 3-kinase (PI3K)/Akt signaling to the actin cytoskeleton. J Biol Chem 2011, 286(34):29758-29770.

24. Springer TA: Traffic signals for lymphocyte recirculation and leukocyte emigration: the multistep paradigm. Cell 1996, 76:301-314.

25. Steeber DA, Tedder TF: Adhesion molecule cascades direct lymphocyte recirculation and leukocyte migration during inflammation. Immunol Res 2000, 22:299-317.

26. Willinger T, Flavell RA: Canonical autophagy dependent on the class III phosphoinositide-3 kinase Vps34 is required for naive T-cell homeostasis. Proc Natl Acad Sci U S A 2012, 109(22):8670-8675.

27. Glynn DJ, Hutchinson MR, Ingman WV: Toll-like receptor 4 regulates lipopolysaccharide-induced inflammation and lactation insufficiency in a mouse model of mastitis. Biol Reprod 2014, 1(90(5)):91. 
28. Koudande DO, Thomson PC, Bovenhuis H, Iraqi FA, Gibson J, van Arendonk J: Biphasic survival analysis of Trypanotolerance QTL in mice. Heredity 2008, 6:407-414.

29. Aylor DL, Valdar W, Foulds-Mathes W, Buus RJ, Verdugo RA, Baric RS, Ferris MT, Frelinger JA, Heise M, Frieman MB, Gralinski LE, Bell TA, Didion JD, Hua K, Nehrenberg DL, Powell CL, Steigerwalt J, Xie Y, Kelada SN, Collins FS, Yang IV, Schwartz DA, Branstetter LA, Chesler EJ, Miller DR, Spence J, Liu EY, McMillan L, Sarkar A, Wang J, et al: Genetic analysis of complex traits in the emerging collaborative cross. Genome Res 2011, 21:1213-1222.

30. Philip VM, Sokoloff G, Ackert-Bicknell CL, Striz M, Branstetter L, Beckmann MA, Spence JS, Jackson BL, Galloway LD, Barker P, Wymore AM, Hunsicker PR, Durtschi DC, Shaw GS, Shinpock S, Manly KF, Miller DR, Donohue KD, Culiat CT, Churchill GA, Lariviere WR, Palmer AA, O'Hara BF, Voy BH, Chesler EJ: Genetic analysis in the collaborative cross breeding population. Genome Res 2011, 2011:1223-1238.

31. Mathes WF, Aylor DL, Miller DR, Churchill GA, Chesler EJ, de Villena F, Threadgill DW, Pomp D: Architecture of energy balance traits in emerging lines of the Collaborative Cross. Am J Physiol Endocrinol Metab 2011, 300:E1124-E1134.

32. Kelada SN, Aylor DL, Peck BC, Ryan JF, Tavarez U, Buus RJ, Miller DR, Chesler EJ, Threadgill DW, Churchill GA, Pardo-Manuel De Villena F, Collins FS: Genetic analysis of hematological parameters in incipient lines of the Collaborative Cross. G3 2012, 2:157-166.

33. Mathes W, Aylor D, Miller D, Churchill G, Chesler E, De Villena FP, Threadgill DW, Pomp D: Architecture of energy balance traits in emerging lines of the Collaborative Cross. Am J Physiol 2010, 300:1124-1134.

doi:10.1186/1471-2164-15-865

Cite this article as: Vered et al: Susceptibility to klebsiella pneumonaie infection in collaborative cross mice is a complex trait controlled by at least three loci acting at different time points. BMC Genomics 2014 15:865.

\section{Submit your next manuscript to BioMed Central and take full advantage of:}

- Convenient online submission

- Thorough peer review

- No space constraints or color figure charges

- Immediate publication on acceptance

- Inclusion in PubMed, CAS, Scopus and Google Scholar

- Research which is freely available for redistribution 